International Journal of Electrical and Power Engineering 12 (2): 6-14, 2018

ISSN: 1990-7958

(C) Medwell Journals, 2018

\title{
A Review of the IEC 61850 Protocol and its Implications for Substation Communication
}

\author{
N.B. Sparks and A.K. Saha \\ Howard College Campus, University of KwaZulu-Natal, King George V Ave, \\ 4001 Durban, South Africa
}

\begin{abstract}
This study presents a review of the IEC 61850 substation protocol and its implications for communication in both modern and legacy-based protection schemes. The intelligent substation devices of today have the ability to communicate, interface and interoperate using ethernet-based protocols like the IEC 61850 standard. This has promoted an industry shift from the outdated serial and legacy protection schemes which rely on binary and analog hardwire connections between device to an ethernet-based architecture. Therefore, the IEC 61850 protocol affects the way substations are designed how IEDs are interfaced, the manner in which devices communicate and the overall effectiveness and efficiency of a substation. However, this new standard also presents a fresh set rules to engineers and utilities alike. These define the manner in which IEDs are configuration as well as how they communicate with other devices using a newly established peer to peer message system. Thus, the continued collaboration of the International Electrotechnical Commission has resulted in the development of a communication standard that allows IEDs of different manufacturers to interact within the local substation environment and transfer data and signals quickly over a 'virtual' ethernet-based network.
\end{abstract}

Key words: Architecture, communication, ethernet, hardwire, IED, IEC 61850, legacy, protection, protocol, relay, standard

\section{INTRODUCTION}

The word 'protection' in the context of this study, refers to the ability of a device to detect a potentially dangerous electrical anomaly such as fault current and immediately or otherwise isolate the vulnerable components of a power system (Blackburn and Domin, 2006; Valdes et al., 2015). The failure of a piece of protective equipment such as a relay to operate under fault conditions can cause system outages, damage to expensive equipment as well as danger to human life (Blackburn and Domin, 2006; Valdes et al., 2015). The design of protection for power systems and substations worldwide has continued to change, develop and evolve as technology improves (Lundqvist and Einarsson, 2007). In times past, the numerous media, mechanisms and methods of communication between equipment within substations has presented an economic and technical obstacle to engineers, vendors and utilities on a global scale (Lundqvist and Einarsson, 2007). Over time relays have become more advanced and complex with many different functions incorporated onto one physical device. As a result, countless protocols have been developed by vendors to help transfer pieces of information between these relays (Hou and Dolezilek, 2010). Therefore, a variety of different proprietary protocols may exist on a network of advanced relays to serve many different functions (Hou and Dolezilek, 2010). However, complex combinations of these protocols can make designing a Substation Automation System (SAS) and integrating devices from different manufacturers a tedious task (Hou and Dolezilek, 2010). A new standard of communication from the International Electrotechnical Commission (IEC) was needed to make the protection, control and automation of a substation more efficient and generic within its local environment.

Modern relays are typically intelligent microprocessor-based devices that are in the most basic sense, used to control and trip electrical circuit breakers upon sensing, for example, a fault current at a particular point on a system (Blackburn and Domin, 2006; Valdes et al., 2015; Lundqvist and Einarsson, 2007). In addition, these intelligent relays have different levels of time-response that enable them to clear abnormalities like under and over-current, under and over voltage and fluctuations in the grid frequency before any damage is

Corresponding Author: N.B. Sparks, Howard College Campus, University of KwaZulu-Natal, King George V Ave, 4001 Durban, South Africa 
incurred on a system (Valdes et al., 2015). Hence, when a fault occurs and an abnormal electrical quantity is sensed, a trip logic is created by the relay and sent to a circuit breaker which interrupts the flow of power to the connected equipment preventing any damage and or other related consequences within the appropriate timeframe (Valdes et al., 2015). These newer, faster and more intelligent relays have now begun to use TCP/IP ethernet-based protocols like the IEC 61850 communication standard which is hence, forth replacing the old ethernet, serial and legacy-based protection schemes (Valdes et al., 2015; Lim and Sidhu, 2013).

Today's relays are often referred to as cyber-physical devices, since, their design is both digital and programmable in nature (Valdes et al., 2015). They have been developed to perform many advanced peripheral functions that act to improve the security and reliability of a power system (Blackburn and Domin, 2006; Hou and Dolezilek, 2010). These functions may include metering, automation, protection, fault finding and recording as well as control and monitoring (Hou and Dolezilek, 2010). Hence, such relays are commonly referred to as Intelligent Electronic Devices (IEDs) (Hou and Dolezilek, 2010). In times past, IEDs communicated using their very own proprietary TCP/IP, serial or hardwire-based communication systems (Hou and Dolezilek, 2010). This posed a technical barrier to both utilities and engineers alike. However, the IEC 61850 protocol allows existing relays to communicate with their neighbouring IED's or to a master control unit within the substation regardless of the manufacturer or vendor (Valdes et al., 2015; Hou and Dolezilek, 2010). The IEC 61850 standard defines the layer, method and protocol for the communication between relays and other IEDs (Hou and Dolezilek, 2010). This allows them to interoperate with other IEC 61850 compliant pieces of equipment, tools and systems. It also introduces new data objects and formats as well as a substation configuration language, commonly referred to as SCL (Valdes et al., 2015; Hou and Dolezilek, 2010). This protocol has been instrumental in the development of 'smarter grids' which is resulting in the subsequent and swift death of legacy, serial and other less favourable TCP/IP protocol-based protection schemes (Valdes et al., 2015). The IEC 61850 standard has allowed engineers to consider innovative designs for smarter substations and to repurpose old equipment within the confines of this new protocol (Valdes et al., 2015; Lundqvist and Einarsson, 2007). The manufacturers of IEC 61850-based IEDs now offer gateways or communication shells that allow the modern IEDs of today to interface and connect to present and or older legacy-based systems (Lundqvist and Einarsson, 2007). This has resulted in the proliferation of 'hybrid' substations.
Historically, each of the protection, control and monitoring devices within a substation required different proprietary communication links in order to transfer important data. This means that communication systems relied on networks of relays using links with EIA-232 point to point and EIA-485 multi-drop communication ports (Hou and Dolezilek, 2010). The speed of data transfer and information exchange over these links was around $38.4 \mathrm{kB} / \mathrm{sec}$ (Hou and Dolezilek, 2010). Two of the communication protocols that were used included DNP and modbus which over time also migrated to the TCP/IP ethernet-based communication medium (Hou and Dolezilek, 2010; Gupta, 2008). Alternatively, the new IEC 61850 standard defines several different interfaces that can be used to communicate between equipment within the substation using the shared physical connections, ethernet links and the substation Local Area Network (LAN) (Lim and Sidhu, 2013; Gupta, 2008). The ethernet communication architectures on which the IEC 61850 protocol is based can be either 10 or $100 \mathrm{Mbit} / \mathrm{sec}$ for the low to high speed processing or transfer of information (Lundqvist and Einarsson, 2007; Hou and Dolezilek, 2010). This makes IEC 61850 much faster than EIA-232 or EIA-485 based protection schemes (Lundqvist and Einarsson, 2007; Hou and Dolezilek, 2010). Hence, forth, the interconnection of IEDs is now standardized regardless of the manufacturer or vendor. This has had significant cost and maintenance advantages over older legacy protection systems that require mostly hardwire-based techniques to connect and interface between different relays based on a physical response from fuse elements and induction disks (Valdes et al., 2015). Therefore, the IEC 61850 communication standard defines the future of substation automation, monitoring, control and protection.

\section{MATERIALS AND METHODS}

Background: The IEC 61850 communication protocol allows IEDs from different vendors to communicate with each other as well as with other devices within the automated substation architecture using a generic, beneficial and commonly understood method of communication. This protocol forms part of the IECs Technical Committee 57 (TC57) which exists to address the reference architecture for power systems. The current and developing communication media include web and internet based services as well as data, information and signal communication over an ethernet or optical fiber-based substation local area network. The message classes that this protocol continues to offer include packets like: client-server messaging, Manufacturing 
Message Specification (MMS), Generic Object-Oriented Substation Event messaging (GOOSE) and Sampled Measured Values (SMV or SV) (Valdes et al., 2015; Lundqvist and Einarsson, 2007 Hou and Dolezilek, 2010). Therefore, the IEC 61850 standard and the associated communication networks can run over TCP/IP-based networks using high speed ethernet communication links which deliver acceptable response times of $<4 \mathrm{msec}$ (Hou and Dolezilek, 2010). This makes the IEC 61850 protocol ideally suited for the fast form of communication that is required during protective relaying (Hou and Dolezilek, 2010).

A brief history: The very first microprocessor distance relays with fault location detection, appeared in the early 1980's (Hou and Dolezilek, 2010). Over the years many protocols have been developed for substationcontrol, protection and automation, some of which have their very own unique communication links and proprietary systems (Hou and Dolezilek, 2010). The IEC 61850 standard offers a of interoperation and intercommunication between IEDs from different manufacturers which is of significant advantage to engineers that specialise in substation automation, protection and design (Valdes et al., 2015). An IEC task team of around 60 group members from various nations around the world developed three IEC working committees in the year 1995. These working groups responded to and identified all the concerns of power utilities, engineers and vendors alike and created the IEC 61850 protocol which made its formal appearance in early 2003. Today, the standard is still enjoying rapid development, growth and implementation worldwide. The objectives that were set by the commission were to:

- Develop a single universal protocol for transporting information, data and signals

- Define the services required to transfer data, signals and information between different and similar devices

- Promote the interoperability between IEDs of different manufacturing vendors within an electrical utility

- Develop a common format for storing information

- To define and specify the types and methods of protection, control and automation testing for the new IEC 61850 protocol

Applications and benefits: A conventional substation is a hardwired-based environment using mostly copper based media to connect tools, hardware and equipment (Jonssen and Apostolov, 2008). Therefore, converting this type of infrastructure into an IEC 61850-based TCP/IP architecture can be considered tedious.If protection equipment and or other devices were to be installed in a conventional substation it then becomes very difficult and costly to move them (Janssen and Apostolov, 2008). So, functions that are practically impossible for legacy-based substations become far easier and more achievable in an IEC 61850 ethernet-based environment where the operation and maintenance of IEDs is more 'virtual' (Valdes et al., 2015; Hou and Dolezilek, 2010; Janssen and Apostolov, 2008). One of the major advantages of the protocol includes the use of functions. All of the functions in a substation are modelled using constructs called Logical Nodes (LN) (Lundqvist and Einarsson, 2007; Mickiewicz, 2006; Janssen and Apostolov, 2008). Therefore, the functions specific to an IED are communicated using Lns which ensures that the communication and exchange of data, signals and information happens in a format that other IEDs can understand (Lundqvist and Einarsson, 2007; Janssen and Apostolov, 2008). Since, the IEC 61850 standard is so, convenient, effective and efficient in its approach to substation automation, protection and control the advantages of this protocol are boundless. The benefits of the IEC 61850 standard are listed as follows (De Mesmaeker et al., 2005):

- Reduced hardwire connection within substations

- Installation of IEDs is not as labour intensive

- Lower maintenance and cheaper commissioning costs

- Facilitates the optimisation of substation architecture

- Functions and capabilities that help to eliminate current transformer saturation and open circuit

- Intercommunication between devices from different vendors

- The inception of a single unified substation protocol

The IEC 61850 standard is a relatively new protocol that is already enjoying widespread popularity within substations for protection and automation due to its advances in communication, configuration, interoperability and topology (Valdes et al., 2015; Lundqvist and Einarsson, 2007). However, one of the major disadvantages of this protocol is that its infrastructure is vulnerable to cyber-attacks simply because it is an ethernet-based architecture (Valdes et al., 2015). IEC 61850-based substation IEDs are programmable, computerized and connected to a substation local area network and even to the internet. So, IEDs rely on these virtual systems for the transfer of cyber information. Therefore, IEC 61850 systems are susceptible to cyber-attacks, since, they are defined as a "hackable" TCP/IP-based infrastructure (Valdes et al., 
2015). Since, this protocol is reasonably new, the applications and potential of the standard are still developing at the hands of the IEC, engineers and utilities alike. The present focus of this protocol is to effectively communicate (Valdes et al., 2015; Gupta, 2008):

- $\quad$ SVs for current and voltage transformers (CT and VT)

- Input/output information and data for control monitoring and protection

- $\quad$ Trip signals and GOOSE messaging

- Configuration information and setup files

- Transfer data to control hubs

- As well as system metering

\section{RESULTS AND DISCUSSION}

Communication classes: The IEC 61850 protocol provides a standardized means of communication for IEDs within a substation. Not only does this standard specify the medium of communication whether optical fibre or ethernet but it delivers a set of functions, formats and layers that define how information or signals are transferred between devices (Valdes et al., 2015; Lundqvist and Einarsson, 2007; Hou and Dolezilek, 2010). In addition to client-server messages, there are two common classes of communication for protection, control and automation, namely: The GOOSE and SV message classes, respectively (Valdes et al., 2015; Lundqvist and Einarsson, 2007; Hou and Dolezilek, 2010). Firstly, client-server integration deals with the services that are needed or used by the Client in order to receive and store information from devices as well as send control signals to the IED servers (Valdes et al., 2015; Lundqvist and Einarsson, 2007; Hou and Dolezilek, 2010). Therefore, a typical client-server system may refer to the Supervisory Control and Data Acquisition (SCADA) or Human
Machine Interface (HMI) of the broader substation importantly, client-server messages are sent through the TCP/IP stack where no specific time constraints are implied (Hou and Dolezilek, 2010).

On the other hand, the GOOSE and SV message classes are exclusively used as packets for sending signals and data between IEDs. These are both real time messages that by pass the TCP/IP stack and interface onto the ethernet link layer (Hou and Dolezilek, 2010). The GOOSE and SV message classes are both vital aspects of the IEC 61850 protocol and were discussed further as follows in this studyof the study (Valdes et al., 2015; Lundqvist and Einarsson, 2007; Hou and Dolezilek, 2010; Carvalheira and Coronel, 2014; Kriger et al., 2013; Anonymous, 2011, 2017). Figure 1 shows how these classes map directly onto the ethernet link layer.

Generic substation events: The two most commonly used horizontal message classes in IEC 61850-based substations are the GSSE and GOOSE message classes, respectively (Valdes et al., 2015; Lundqvist and Einarsson, 2007; Hou and Dolezilek, 2010). The major difference between them is that GOOSE can transfer data formats like analog, binary and integer information whereas GSSE is constrained mainly to binary event status data (Carvalheira and Coronel, 2014; Kriger et al., 2013; Anonymous, 2017). These classes are both referred to as peer to peer communication mechanisms that transfer information in a 2-layer message between the bay and process levels (Carvalheira and Coronel, 2014; Kriger et al., 2013; Anonymous, 2017). GOOSE messages allow for the transfer of a vast range of common data that is organized by a DATA-SET (Kriger et al., 2013). Hence, this message system is favoured, widely understood and enjoys greater popularity within IEC 61850-based

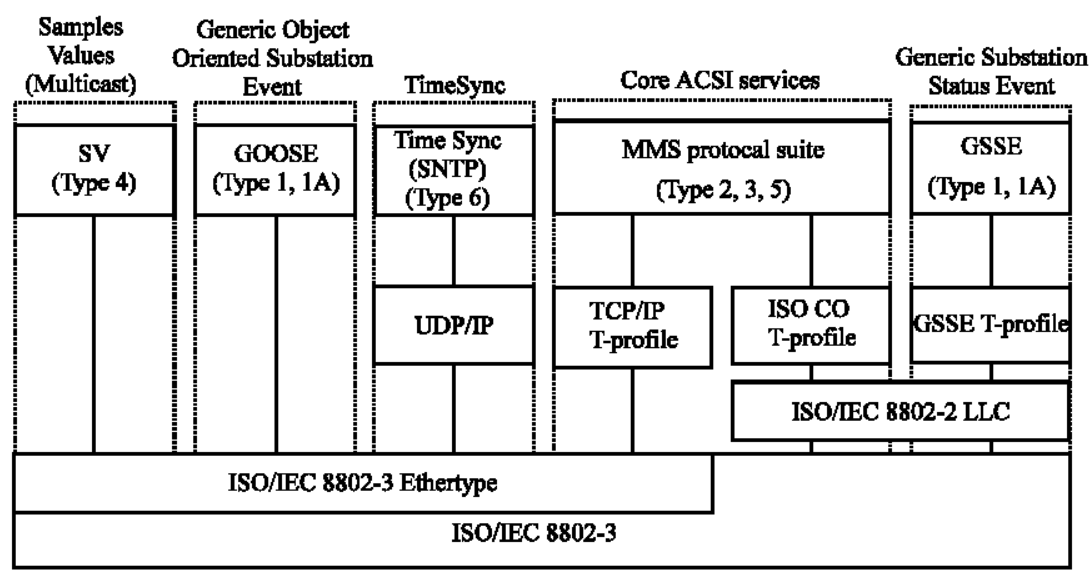

Fig. 1: IEC 61850 message class data mapping 
Int. J. Elec. Power Eng., 12 (2): 6-14, 2018

environments due to its greater flexibility. GOOSE messages are typically used by IEDs to report status events to other IEDs within and between feeders. It was developed to operate on TCP/IP ethernet or optical fibre networks to replace the old hardwire or serial communication links between IEDs and legacy relays on the station bus (Valdes et al., 2015; Lundqvist and Einarsson, 2007; Hou and Dolezilek, 2010). To setup an IEC 61850 communication network is a fairly simple procedure. Each IED within a network will have an IP address which will specify which GOOSE messages the device has access to. This makes the GOOSE message system and by extension IEC 61850 more efficient than legacy-based architectures. In addition to its flexible transfer of data, the GOOSE message class is also configurable and can send information like circuit breaker status and analogue measurements. This means that a particular IED could issue a GOOSE message to its peer devices notifying them if it had issued a trip signal (Hou and Dolezilek, 2010). The applications of this allow the IED closest to the fault (the downstream IED) to issue a trip and at the same time ensure that the other upstream IEDs do not trip needlessly, thus reducing the size of the outage (Hou and Dolezilek, 2010; Carvalheira and Coronel, 2014). This is called a blocking response or sympathetic trip protection (Hou and Dolezilek, 2010). Therefore, IEDs connected to the substation LAN are aware of the currents and voltages at the points that they measure directly as well as from the GOOSE messages that are received from the other IEDs (Valdes et al., 2015) (Hou and Dolezilek, 2010; Carvalheira and Coronel, 2014.

Typically, a number of relays may be used to take readings on a particular line or bus section with GOOSE messages being exchanged between those devices who have subscribed to the information stream (Hou and Dolezilek, 2010). Importantly, GOOSE messages include items like trip, interlocking and inter-trip messages (Valdes et al., 2015; Hou and Dolezilek, 2010 ; Kriger et al., 2013. These messages are time critical and must be transmitted at speeds of between 10 and $3 \mathrm{msec}$ (Anonymous, 2011). This means that GOOSE is referred to as a 'fast' messages system and is used to decrease the clearing time of faults. Usually, no more than $4 \mathrm{msec}$ is allowed to elapse from the time a particular event in the system is detected until the point at which the message is transmitted (Hou and Dolezilek, 2010). As previously mentioned, GOOSE messages are multi-cast to the substation LAN and are only accepted by those IEDs that have been configured to subscribe to that particular data stream (Hou and Dolezilek, 2010). Importantly, these messages are broadcast multiple times using a $3 \mathrm{msec}$ back-to-back retransmission mechanism that transmits

\begin{tabular}{llll}
\multicolumn{4}{l}{ Table 1: Time performance and message types (Hou and Dolezilek, 2010) } \\
\hline \multicolumn{1}{l}{ Type } & Application & Class & Transmission (msec) \\
\hline 1A & Fast messages & P1 & 10 \\
& (Trip) & P2/P3 & 3 \\
1B & Fast messages & P1 & 100 \\
& (Other) & P2/P3 & 20 \\
2 & Medium speed & & 100 \\
3 & Low speed & & 500 \\
4 & Raw data & P1 & 10 \\
& & P2/P3 & 3 \\
5 & File transfer & & $\geq 1000$ \\
6 & Time sync & & (Accuracy) \\
\hline
\end{tabular}

regardless of whether a change has occurred (Hou and Dolezilek, 2010). This improves the reliability of the system and ensures that the event has been received by the appropriate IED (Valdes et al., 2015; Lundqvist and Einarsson, 2007; Hou and Dolezilek, 201 0; Anonymous, 2017). Therefore, GOOSE messages are an example of multicast/broadcast messages i.e., a single device will send out a message to several devices in the multicast case and all the devices on the network in the broadcast case (Hou and Dolezilek, 2010). This has huge benefits over the point-to-point message systems of old. When the ethernet switch receives a particular message, it forwards it to all other ports on the network apart from the port where the message was received (Valdes et al., 2015; Lundqvist and Einarsson, 2007; Hou and Dolezilek, 2010). The IED must then analyse this message and decide whether or not it has been configured to receive it. This constricts the bandwidth of the network and can increase network traffic affecting the speed of the messages that are transmitted. Two methods exist to alleviate this problem. One is to develop a system of several virtual LANs that divide the traffic between them (Lundqvist and Einarsson, 2007; Hou and Dolezilek, 2010; Carvalheira and Coronel, 2014; Anonymous, 2017). The other solution is to use the fact that GOOSE messages may be assigned a priority, so that, the switch knows which messages to send first. The time duration and speed of transfer of messages in the IEC 61850 standard are shown in Table 1 (Hou and Dolezilek, 2010). The table specifies that GOOSE messages are a 1 or $1 \mathrm{~A}$ fast message system (Hou and Dolezilek, 2010).

Sampled values: The GOOSE and SV message classes are both referred to as priority real-time messaging that can interface directly over the substation LAN. The SV message class is used to send digitized voltage and current measurements to the IEDs within the substation on the process bus (Valdes et al., 2015; Lundqvist and Einarsson, 2007; Hou and Dolezilek, 2010; Kriger et al., 2013; Anonymous, 2011, 2017). SV messages are also multicast which means that the data obtained from a measurement taken at one location may 


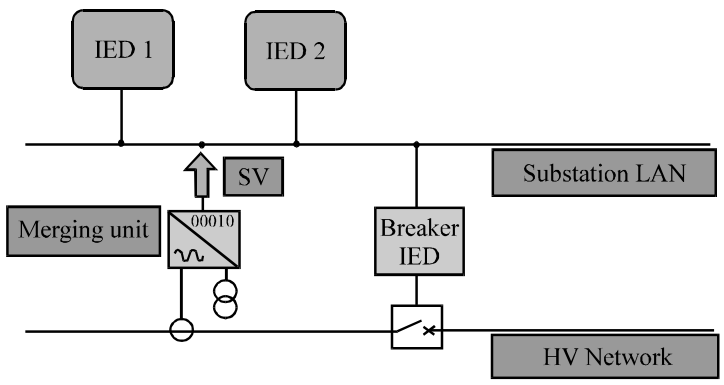

Fig. 2: IEC $61850 \mathrm{SV}$ messaging and MU interfaces (Carvalheira and Coronel, 2014)

be sent to any number of devices connected to the ethernet network. This measured information is typically obtained from the secondary analog outputs of the instrument CTs and VTs respectively (Hou and Dolezilek, 2010). Furthermore, the analog data from the instrument transformers must be sent to a device called a Merging Unit (MUs) whose function is to digitize the sensed information and finally deliver it to the IEDs using logical interfaces '4'and ' 5 ' (Janssen and Apostolov, 2008). Modern IEDs can input different data from multiple MUs on the network. The MU can take 80 samples per cycle with an $\mathrm{SV}$ message rate of $4.8 \mathrm{kHz}$ for basic protection and 256 samples per cycle for high frequency applications other SV message rates may also include $1.5,4$ and $12 \mathrm{kHz}$, respectively (Valdes et al., 2015; Lundqvist and Einarsson, 2007; Hou and Dolezilek, 2010). Therefore, a merging unit is a device that provides the interface between the CTs/VTs and the IEDs within a substation and they can receive multiple binary and analog inputs (Valdes et al., 2015; Hou and Dolezilek, 2010; Anonymous, 2017). Figure 2 shows a basic implementation of this concept (Carvalheira and Coronel, 2014).

Additionally, the IEC 61850 protocol also allows relays to send messages via. the $\mathrm{MU}$ to other devices on the station bus. Hence, the MU may arbitrate the interaction between a certain number of IEDs and communicate with other MU's connected to the substation LAN (Valdes et al., 2015; Hou and Dolezilek, 2010). An IED must, however, be configured to subscribe to the SV stream from its associated MU and can then also receive GOOSE messages and phasor information from its surrounding IED's provided it is configured to do so. It is common that several merging units interface with one another over the substation LAN which can transfer information at speeds of up to $100 \mathrm{MB} / \mathrm{sec}$ (Lundqvist and Einarsson, 2007; Hou and Dolezilek, 2010). Numerous devices can be interconnected using this system as well as a central supervisory computer which may receive current and voltage samples in the form of SV messages from the MU (Lundqvist and Einarsson, 2007). This means that substation protection, control and monitoring can be implemented in a very coherent fashion.

Hence, the IEC 61850-based communication architecture may have an impact on the design of substations and on the layout of switchgear as compared to legacy-based protection schemes (Valdes et al., 2015; Hou and Dolezilek, 2010). In summary, MU's carry the following functionalities:

- Signal processing of sensors

- Synchronisation of three-phase voltage and current measurements

- Analog interfacing

- $\quad$ Digital Interfacing (IEC 61850-9-2)

Logical interfaces: One of the most important benefits of the IEC 61850 protocol lies in the ability of relays or IEDs to react in a collaborative fashion when dealing with an electrical anomaly like fault current (Gupta, 2008). The IEC 61850 standard allows the IED's, that sense items like fault current and trip electrical circuit breakers to inform their peer relays of the protective actions that were undertaken or needed using the shared physical communication links between physical devices (Gupta, 2008). Thus, a function used by a particular IED may be specific to that device such as an IED on a particular feeder or distributed to two or more IED's over the ethernet communication network if they subscribe to that particular information stream. Thus, the allocation of functions between IED's in a substation defines the requirements of the aforementioned physical interfaces.

All known functions within a substation may be modelled using Logical Nodes (LNs) (Gupta, 2008). There are logical nodes for automatic control whose names begin with ' $\mathrm{A}$ ' as well as logical nodes for Protection (P) and Monitoring (M) as well as nodes for all other necessary function within the substation. Each node has an instance-ID as a suffix to delineate between nodes as they are transferred thus, ensuring that LNs are not received by the incorrect IED (Janssen and Apostolov, 2008). These nodes communicate with each other and transfer information using logical interfaces. The logical interfaces within a substation distribute IED 1 IED $2 \mathrm{HV}$ network substation LAN CB CB Merging Unit SV functions (logical nodes)between IEDs on different levels of the substation hierarchy (station, bay and process buses) from IF1-IF10 as shown in Fig. 3 (Gupta, 2008; Janssen and Apostolov, 2008). Therefore, IF 1-10 are referred to as logical function interfaces and represent the 
Int. J. Elec. Power Eng., 12 (2): 6-14, 2018

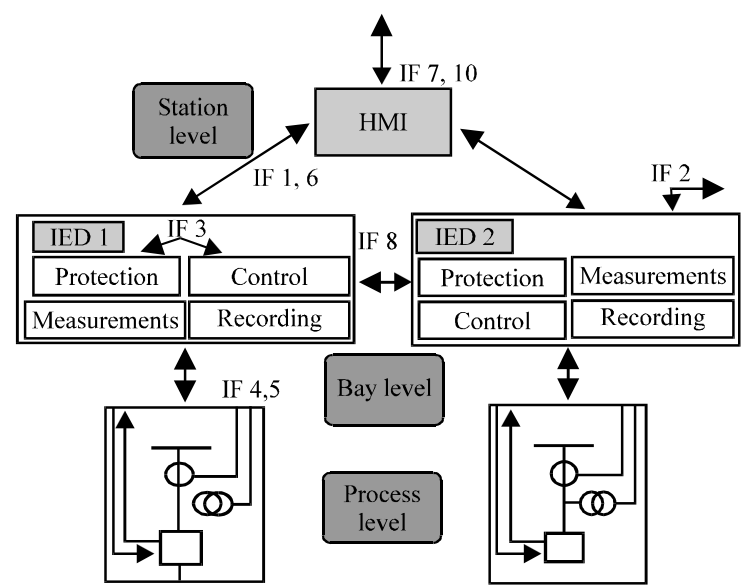

Fig. 3: IEC 61850 logical interfaces (Janssen and Apostolov, 2008)

different hierarchies over which specific information like trips, inter-trip and interlocks can be transferred between various IEDs in a substation. The researchers of study (Janssen and Apostolov, 2008) define the IF4 and IF5 functional levels, shown in Fig. las interfaces for the transfer of data from the CTs and VTs between the process level and the bay level, respectively (Janssen and Apostolov, 2008). Alternatively, the IF8 functional level refers to the direct transfer of data between bays for fast information processes like interlocking (Janssen and Apostolov, 2008). IF 4 and 5 are typically used for process bus applications whereas IF 8 has applications for station bus communications. The logical function interfaces that link devices and IEDs from IF1 up to IF10 were shown graphically in Fig. 3 (Janssen and Apostolov, 2008).

Substation configuration language: The IEC 61850 protocol specifies that engineers and vendors use a Substation Configuration Language (SCL) which was developed to configure the settings and format the functions within a particular IED. Manufacturer specific IED configuration tools are used to convert the functionality, communication mechanisms and the parameters of an entire IED into a hierarchy of SCL system files. This is one of the major differences between IEC 61850-based IEDs and other protocol-based substation automation systems. SCL is a description configuration vernacular that is based on eXtensible Markup Language (XML). SCL specifies a file format that describes IED communication, switch yard structure and any relations that take place between them. It ensures that the IED Capability Descriptions (ICDs) and the substation descriptions are transferred between the IED engineering tools and the system engineering tools of different vendors or manufacturers. Upon setup, the IED configurator tool converts functionality, data communication, events and alarms into SCL (Lundqvist and Einarsson, 2007; Lim and Sidhu, 2013). This simplifies substation configuration and communication between IEDs. Therefore, the generation of data, reading of data and data structure is less ambivalent (Lundqvist and Einarsson, 2007; Lim and Sidhu, 2013).

In addition, SCL specifies file formats that describe the configuration, parameters and the relationship between IEDs. Furthermore, SCL engineering results in the creation of a Substation Configuration Description file (SCD) which contains all the necessary information about a substation (Lundqvist and Einarsson, 2007; Lim and Sidhu, 2013). As previously mentioned, SCL allows the easy and simple exchange of information between IEDs of different manufacturers. Hence, when a new IED is installed within a substation the configuration of the previous IED is available in SCL format and can simply be imported onto the new device. This concept is similar to changing a SIM card in a new phone (Lim and Sidhu, 2013). So, the functionality and communication of the existing protection, automation and control schemes are effectively maintained and hence, SCL engineering has major advantages over older legacy-based systems which are not programmable and must be replaced rather than reconfigured (Lim and Sidhu, 2013). In protection systems, the time-current response of a particular IED can be configured using SCL. The response logic within a particular IED may call for an instantaneous trip when the current exceeds a particular value or a delayed trip where the IED integrates the value of the fault current over time and waits for it to exceed a second threshold value (Lundqvist and Einarsson, 2007). Hence, over current protection may be implemented using SCL in stages.

SCL-based protection: The initialisation and setup of an SCL-based protection IED involves the use of three files, namely: the 'startup.cfg' 'datamap.cfg' and CID files. The function of the first file, called the 'startup.cfg' file is simply to store logical device data. In addition, mapping information is stored in the 'datamap.cfg' file and lastly, the CID file stores the function and role of the IED (Lundqvist and Einarsson, 2007). Furthermore, the protection IED parses a CD file that stores the data which defines the roles that are specific to a particular IED. The IED not only parses these three files but is also initialized via. data object generation, information mapping, network setting, logical node setting and the subscription that allows it to broadcast and receive SV data from the instrument transformers and MUs (Lundqvist and Einarsson, 2007; Lim and Sidhu, 2013). Thus, this means 
that the protection type, function and configuration of the IED is determined by parsing the CID file (Lim and Sidhu, 2013). In order to configure an IEC 61850 relay certain SCL-based communication files are required (Lim and Sidhu, 2013). In summary, these files may include but are not limited to the (Lundqvist and Einarsson, 2007; Lim and Sidhu, 2013):

- System Specification Description file (SSD): which stores information like, logical nodes, physical connections and single-line diagrams of the substation

- System Exchange Description file (SED): stores the information specific to communicating with other configurators

- IED Capability Description file (ICD): which identifies the logical nodes that are available to the IED

- Substation Configuration and Description file (SCD): which describes data exchange and transfer structures and hence, the interaction between IEDs in the system or project

- Configured IED Description file (CID): which enables and configures the IED according to its functions

IED setup using SCL: The first and most fundamental aspect of setting up an IED using SCL is to select a suitable IED for the intended function and or role within the substation and configure it using the IEDs configuration tool. An IED Capability Description (ICD) is produced for each relay based on the IED specific description file. This file describes the logical devices and nodes, GOOSE and SV information, communication services and addresses for data (Lundqvist and Einarsson, 2007; Lim and Sidhu, 2013). In the second step, all the ICD files are transferred to the IED configuration tool. This configures the system functions and allocates functions to the IEDs within the substation (Lundqvist and Einarsson, 2007; Lim and Sidhu, 2013). The system configurator then creates a Substation Configuration and Description file (SCD) after it has received the ICDs, SSD and SED files. The SCD file defines the interaction between different IEDs and holds all the information that describes the substation. Finally, based on this SCD file an IED engineering tool is used to build the CID file for each device (Lim and Sidhu, 2013). The system configurator then sends the IEDs their specific CID files. The IEDs parse these files during the initial start-up and the configuration is complete. The file configuration system described above was depicted in Fig. 4.

Benefits of SCL: SCL engineering is used by engineers and utilities to best suit the user's requirements and by extension the requirements of the substation. SCL allows

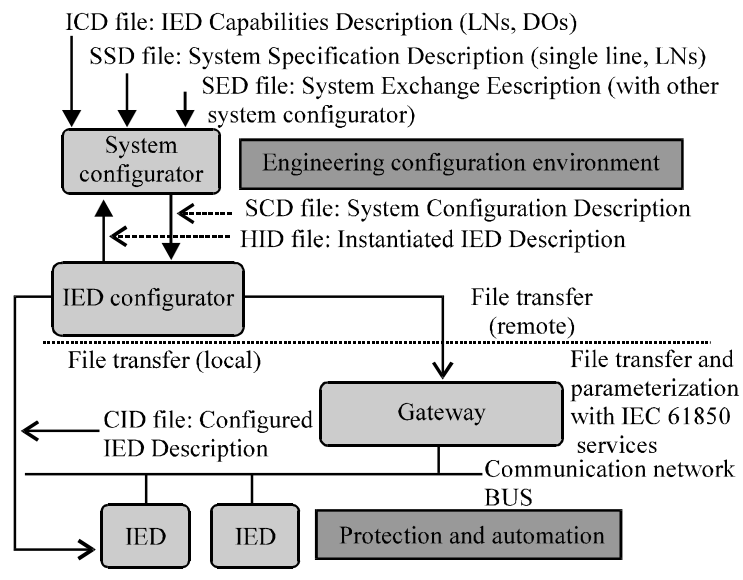

Fig. 4: SCL file transfer (Lundqvist and Einarsson, 2007)

offline configurator tools to produce the necessary files needed for the configuration of IEDs automatically, purely based on the specifications of the designed power system. This means that there need not be a connection to the IED network for IED client configuration. This has significant cost advantages and eliminates the manual labour required for manual IED configuration tasks. In addition to setup, SCL also allows the distribution of IED configurations among vendors which makes this language somewhat generic.

\section{CONCLUSION}

Substation protection, monitoring, automation and control collectively rely on the intercommunication of intelligent devices and equipment.This study presents a comprehensive review of the IEC 61850-based communication architecture and its associated substation related implications. This new communication standard has a marked effect on the way in which data, signals and functions can be shared between IEDs, control centres and other electronic and electrical elements within a substation. Modern relays from companies like SEL, Schneider and $\mathrm{ABB}$ are being developed with this new protocol in mind and can interoperate with other IEDs regardless of the manufacturer or vendor. Therefore, the age of legacy protection systems and conventional substations is at an end. The final standard as specified by the IEC defines a single protocol for modelling various pieces of data within a substation. It seeks to promote the interoperability and communication between IEDs that were developed by different vendors as well as synthesize a common method for storing information. The IEC 61850 standard also provides the basic services needed to transfer data, signals and enable control between IEDs and a central control unit or HMI. Therefore, from the 
thorough investigation conducted in this study it can be concluded that this newly developed ethernet-based protocol is at the forefront of the intercommunication between devices for information and data transfer over the substation LAN. Hardwired electromechanical legacy-based systems are the past, IEC 61850 is the present and the future.

\section{ACKNOWLEDGEMENTS}

The resaerchers of this study wish to acknowledge the researchers of the referenced study and any other contributory works for developing and maintaining the IEC 61850 protocol which was extensively investigated during the course of this submission. Additionally,acknowledgement must be given to the University of KwaZulu-Natal in Durban, South Africa whose facilities and knowledge were used to compile this document.

\section{REFERENCES}

Anonymous, 2011. Substation technical guidebook-IEC 61850 and IEEE 1588 in smart substations. Moxa Europe $\mathrm{GmbH}$, Unterschleißheim, Germany. https://www.moxa.com/Solutions/Substation/eBoo k/index.htm

Anonymous, 2017. IEC 61850 substation overview and smart grids. Moxa Europe $\mathrm{GmbH}$, Unterschleißheim, Germany. https://www.moxa. com/support/request_catalog_detail.aspx?id=1425

Blackburn, J.L. and T.J. Domin, 2006. Introduction and General Philosophies. In: Protective Relaying-Principles and Applications, Blackburn, J.L. and T.J. Domin, (Eds.). Taylor \& Francis, Milton Park, Didcot, UK., pp: 31-58.

Carvalheira, E. and J. Coronel, 2014. Testing the protection system in IEC 61850 communication based substations. Proceedings of the 2014 IEEE International Conference on ANDESCON, October 15-17, 2014, IEEE, Cochabamba, Bolivia, ISBN: 978-1-4799-6686-8, pp: 1-1.
De Mesmaeker, I., P. Rietmann, K.P. Brand and P. Reinhardt, 2005. Substation Automation based on IEC 61850. Proceedings of the 6th International Conference on Cigre SC B5 Regional CIGRE in Cairo, November 21-23, 2005, ABB India, Zurich, Switzerland, pp: 21-23.

Gupta, R.P., 2008. Substation automation using IEC61850 standard. Proceedings of the 15th International Conference on National Power Systems (NPSC), December 16-18, 2008, Indian Institute of Technology Bombay, Mumbai, India, pp: 462-466.

Hou, D. and D. Dolezilek, 2010. IEC 61850-What it can and cannot offer to traditional protection schemes. SEL J. R. Pwr., 1: 1-11.

Janssen, M.C. and A. Apostolov, 2008. IEC 61850 impact on substation design. Proceedings of the 2008 T\&D IEEE/PES International Conference on Transmission and Distribution and Exposition, April 21-24, 2008, IEEE, Chicago, Illinois, USA., ISBN: 978-1-4244-1903-6, pp: 1-7.

Kriger, C., S. Behardien and M.J.C. Retonda, 2013. A detailed analysis of the GOOSE message structure in an IEC 61850 standard-based substation automation system. Intl. J. Comput. Commun. Control, 8: 708-721.

Lim, I.H. and T.S. Sidhu, 2013. Design of a backup IED for IEC 61850-based substation. IEEE. Trans. Power Delivery, 28: 2048-2055.

Lundqvist, B., and B.B.T. Einarsson, 2007. A user friendly implementation of IEC 61850 in a new generation of protection and control devices. ABB. Power Technol. Sweden Rep., 1: 1-6.

Valdes, A., C. Hang, P. Panumpabi, N. Vaidya and C. Drew et al., 2015. Design and simulation of fast substation protection in IEC 61850 environments. Proceedings of the 2015 International Workshop on Modeling and Simulation of Cyber-Physical Energy Systems (MSCPES), April 13, 2015, IEEE, Seattle, Washington, USA., ISBN:978-1-4799-7358-3, pp: 1-6. 